\title{
Light Guide Collector Prototype: Laboratory Testing
}

\author{
Jitka Mohelnikova ${ }^{\mathbf{1}, *}$, Stanislav Darula ${ }^{2}$, \\ Ayodeji Omishore ${ }^{\mathbf{1}}$, Petr Mohelnik ${ }^{\mathbf{1}}$, Denis Miček ${ }^{\mathbf{1}}$ \\ ${ }^{1}$ Faculty of Civil Engineering, Brno University of Technology, Brno, Czech Republic \\ ${ }^{2}$ Institute of Construction and Architecture, Slovak Academy of Sciences, Bratislava, \\ Slovakia
}

Received 25 October 2017, Accepted 21 December 2017

\begin{abstract}
The article reviews the potential of a light guide system equipped by a concentrator device capturing daylight applicable for illumination of building interiors and presents results of experiments on performance of its prototype. The main goal is focused on the comparison of traditional solutions and newly developed prototype of the light guide system and presents examination of its light transmission efficiency based on the laboratory experiments.
\end{abstract}

Keywords: daylighting, tubular light guide, light transmission efficiency.

\section{Introduction}

Light guide system is a device that transmits daylight into building interiors. A function of tubular light guides is to capture, collect and transmit daylight into interior spaces which cannot be lit using conventional windows or skylights where daylight is insufficient and/or is missing [1-4]. These systems compared with traditional skylights and windows show several advantages like energy savings (small envelope area relative to the amount of useful light they can admit), lower summer solar heat gains and daylight presence in interiors. The availability and temporal changes of natural light in internal environment can also contribute to the improvement the occupants' well-being [3].

Systems with daylight collectors are usually placed on the building roof or envelope construction. They capture sunlit or skylight to transmit it into the light tube. The collector installation should be designed in buildings that there is a free space for receiving light while any obstruction of solar radiation from adjacent structures should be avoided. The roof collectors suit the deep parts of building, the façade mounted systems are more suitable for the multi-story buildings, but they are recommended to be south oriented.

The collector function is to gather sunlight and skylight from part or whole sky hemisphere. The light guide is usually covered by a transparent dome to seal the pipe against ingress of dust and rain. The dome is designed to meet the fire resistance requirements. It is self-cleaning due to its shape. The material is a single or multiple-layer transparent glass or plastic in various geometric shapes. It may contain optical features (prisms, reflectors or diffusing elements) to enhance the lighting output, especially at low sun altitude angles [5-7].

Many theoretical and experimental works were focused on the construction of light guides and their light transmittance evaluations [2-4 and 8-17]. Special light guide systems have a head in the shape of the collector to concentrate sun rays and direct them into the light guide tube. A prototype of a light guide with a mirrored parabolic head [18] was tested in the laboratory at the Faculty of Civil Engineering of Brno University of Technology (FCE BUT). Results of the daylight measurements on the reference plane in the experimental box are

\footnotetext{
* Mohelnikova J, Tel.: +420 541147422.

E-mail address: mohelnikova.j@fce.vutbr.cz
} 
summarized in this article.

\section{Light Guide Prototype}

There are several technologies capturing sun rays on the top of buildings and transport them into interiors. Commonly such devices consist of a dome, hollow tube and diffuser. On the top of the tube a transparent cover can be placed. The cover is provided in various shapes or from materials redirecting incident light rays. The light guide with light collecting mirror parabolic head (CMP) was proposed and tested in its prototype. This system can be assembled as stable or equipped by tracker to set direction towards the sun position. Incoming sun rays are reflected on the parabolic mirror. The reflected rays are concentrated in the focus of the mirrored parabola. The secondary mirror located in the focus reflects the concentrated rays towards the light pipe as is shown in Figure 1. The elevations of the sun rays correspond to situations in Brno on 21 ${ }^{\text {st }}$ June at 8:00 (Fig. 1a) and 10:00 (Fig. 1b).

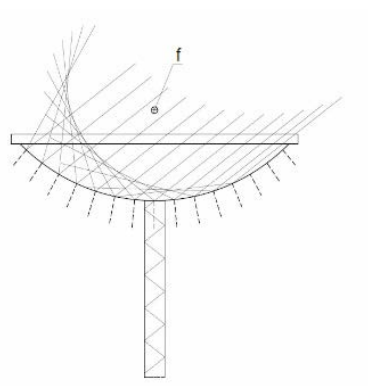

a)

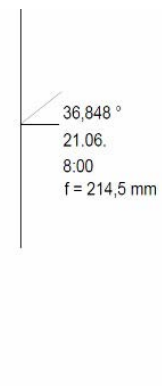

b)

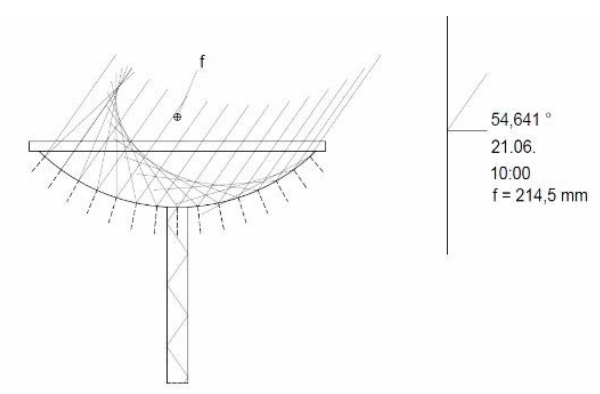

Figure. 1 Scheme of the parabolic head and light rays concentrations on $21^{\text {st }}$ June a) at $8: 00$, solar altitude $36.85^{\circ}$; b) at $10: 00$, solar altitude $54.64^{\circ}$;

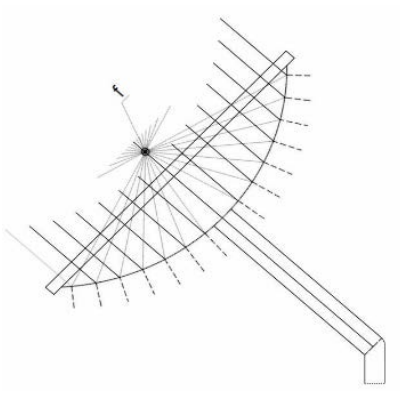

Figure. 2. Scheme of the CMP directed to the sun in elevation $43^{\circ}$

The light pipe with parabolic head is more efficient in hot climate with intensive sun shining when high solar altitudes occur. In temperate climate the system with light concentrator should be tracked to the sun position on the sky. The automatic tracking requires special devices with appropriate software. If tracker is used to direct the CMP towards the sun position than sun rays are parallel to the axis of entry tube as it is shown in Figure 2.

One sample of the CMP prototype was selected for testing of the light transmittance and daylight study. Experiments were carried out with the system consisting of a collector head joined with a tube and diffuser in the static vertical position. It is supposed that the concentrating parabolic mirror located on the building roof can increase light transmittance through the light guide not only due to direct solar radiation but also by diffuse sky light. This will be important finding for the installation of the CMP system in localities with dominant cloudy sky conditions. View into the collecting head is presented in the Figure 3 [18]. The lower part of the collector creates parabolic mirror which reflects sun rays to the upper small metallic concave element with highly reflective surface. The element is used to direct sun rays into the hollow tube with internal surface of the high reflectance. 


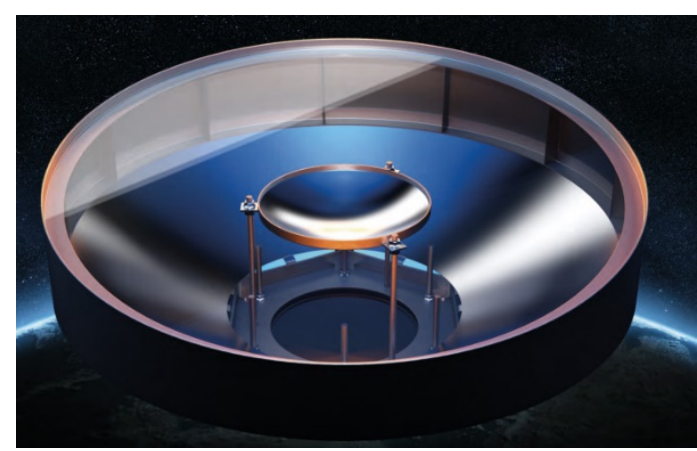

Figure. 3 View onto the CMP head [18]

\section{Laboratory Experiment}

An experimental box was made for the light guide prototype installation. The box is of dimensions $1.0 \mathrm{~m} \times$ $1.0 \mathrm{~m} \times 1.0 \mathrm{~m}$, inside of the box - wooden slab surface without painting. The box serves a load-bearing construction. The sample of the special light guiding system consists of $0.520 \mathrm{~m}$ diameter and $0.6 \mathrm{~m}$ length hollow tube was mounted onto the box. The system includes the CMP collector and a concave element with highly reflective surface, Figure 3. A transparent cover of the collector is made of the clear glass with antireflective coating. The tube end is covered with a translucent diffuser on the indoor side. All joints of the light guide were overlapped by opaque tapes.

Illuminance was measured outside and inside of the box. These measurements give data for specification of light transmission efficiency of the light guiding system. The outside measurements were completed on a horizontal plane at the input of the light pipe concentrator head. The measurements of the horizontal illuminance inside of the box were carried out in the position of the light pipe vertical axis and at the distance of $0.75 \mathrm{~m}$ below the diffuser, Figure 4.

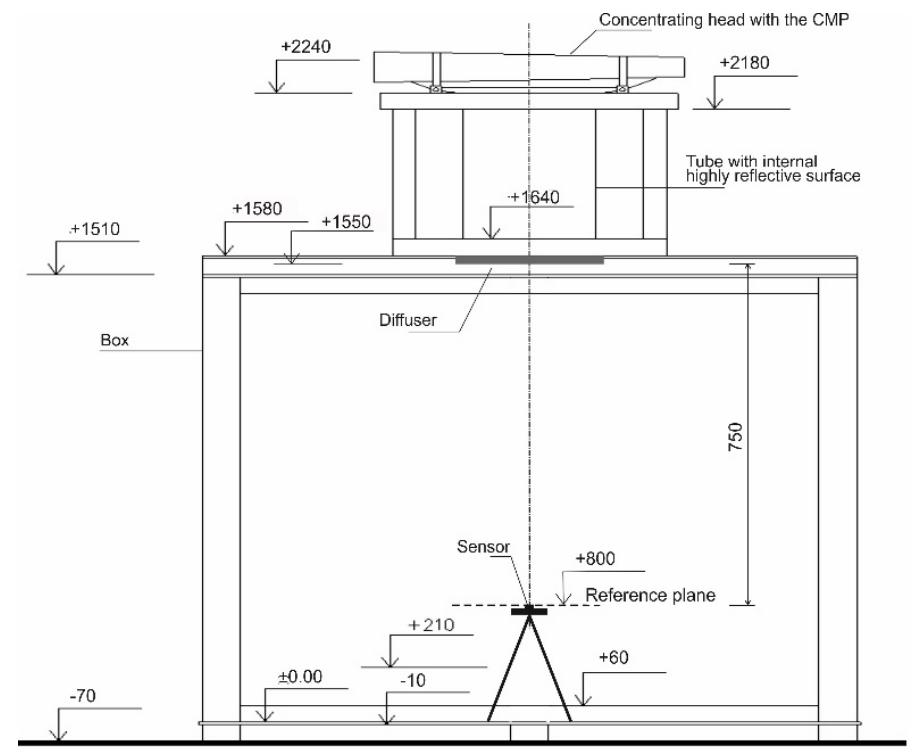

Figure. 4 The experimental setup, location of the light guide with the CMP head and reference plane

The light guide tube was made of hard aluminium sheet coated by high reflective layer on its inner surface. Light transmission efficiency is highest if the tube is short and straight. If tube is longer, bended, or flexible construction, a part of the transmitted light is lost. To minimize light losses, a high reflectivity of the tube lining and glossy inner surface is crucial. This assumption was taken into consideration for the experimental work: light pipe with tube of $0.6 \mathrm{~m}$ length was selected for the testing while the light reflectance of the tube internal surface 
is $\rho=0.93$.

The illuminance meter LX-1128SD was used for light measurements. Illuminances in the investigated points in the interior laboratory and inside of the box were monitored. Daylight conditions were continually registered by the horizontal illuminance meter placed in exterior close the skylight glazing. The measurements were carried out for different daylight levels during a day and under situations from overcast sky to partly cloudy and sunny sky conditions.

\section{Daylight Illuminance Measurement}

Quality of indoor luminous environment depends on the sufficient daylight. Application of new daylight technologies requires testing not only optical properties but also their performance during typical situations.

The prototype of the light pipe with the CMP was used for experimental study and daylighting measurements before starting of its commercial production. Measurement instruments were installed in April 2015 and data were recorded up to July 2015. Daylight illuminance at the light guide input and output serve for specification of the light guide system efficiency for different sky conditions. The measurements were completed inside of the laboratory at the level of the glass cover of the CMP head and inside of the box. Additional measurements of the outdoor daylight illuminance were also simultaneously carried out.

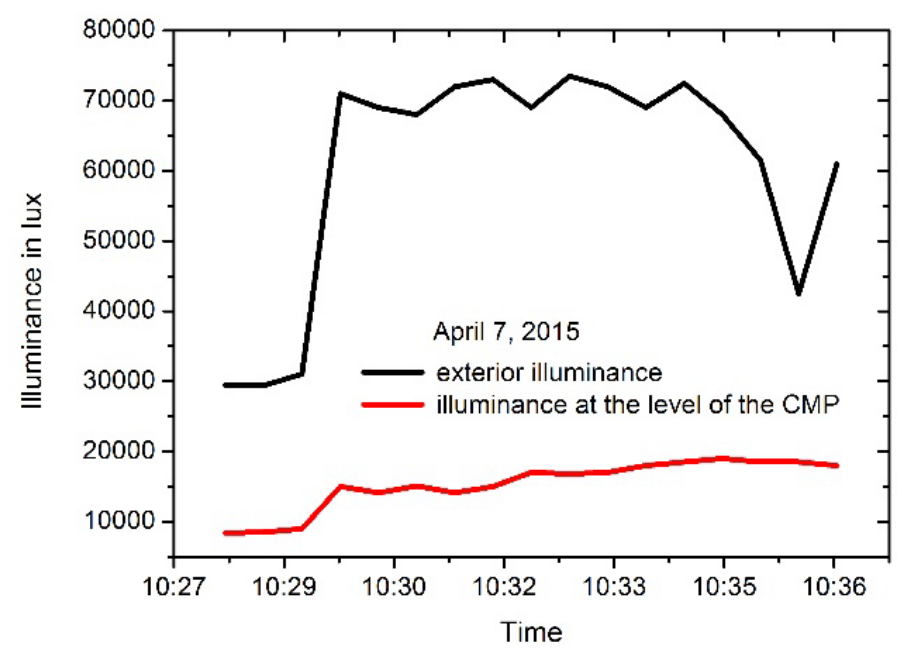

Figure. 5 Fragment of the exterior illuminance and illuminance at the level of the light guide head

Table. 1 Illuminance measured during morning on April 7, 2015 in Brno

\begin{tabular}{ccc}
\hline \multirow{2}{*}{ Time } & Exterior illuminance & Illuminance at the level of the CMP \\
\cline { 2 - 3 } & {$[\mathrm{lx}]$} & {$[\mathrm{lx}]$} \\
\hline $10: 28: 31$ & 29500 & 8400 \\
\hline $10: 29: 01$ & 29400 & 8500 \\
\hline $10: 29: 31$ & 31000 & 9000 \\
\hline $10: 30: 01$ & 71000 & 15000 \\
\hline $10: 30: 31$ & 69000 & 14100 \\
\hline $10: 31: 01$ & 68000 & 15100 \\
\hline $10: 31: 31$ & 72000 & 14100 \\
\hline $10: 32: 01$ & 73000 & 15000 \\
\hline $10: 32: 31$ & 69000 & 17000 \\
\hline $10: 33: 01$ & 73500 & 16800 \\
\hline $10: 33: 31$ & 72000 & 17000 \\
\hline $10: 34: 01$ & 69000 & 18000 \\
\hline $10: 34: 31$ & 72500 & 18500 \\
\hline $10: 35: 01$ & 68000 & 19000 \\
\hline $10: 35: 31$ & 61500 & 18500 \\
\hline $10: 36: 01$ & 42500 & 18500 \\
\hline $10: 36: 31$ & 61000 & 18000 \\
\hline
\end{tabular}


A fragment of a morning illuminance profile of the measurements is shown in Figure 5. The black curve indicates cloudy exterior situation, when illuminance levels change in the range of 29400 to 73000 lx. Horizontal illuminance close the CMP entrance at its level was registered more stable from 8400 to $19000 \mathrm{~lx}$, representing higher diffusivity of incident light, Table 1 . These levels as light source can be used in the study of light transmission efficiency of the sample.

\section{Daylight Luminous Efficiency Modelling}

The luminous flux method applying measured illuminance at the entrance surface area and at the level of the output can be used for the determination of the light transmission efficiency of the light guide systems. Light level - illuminance is the total luminous flux $\Phi_{e}[\mathrm{~lm}]$ incident on a surface per unit area. If illuminance $E_{e}[\mathrm{~lx}]$ at the entrance of the system is known the input luminous flux $\Phi_{e}[\mathrm{~lm}]$ can be calculated [1]. To determine light transmission efficiency $\eta$ of the hollow light guide systems the luminous flux $\Phi_{i}$ [lm] leaving diffuser translucent component in the interior side has to be expressed.

The entrance surface area of the tube is calculated as:

$$
S=\pi \times r^{2} \quad\left[\mathrm{~m}^{2}\right]
$$

The sensor of illuminance meter was fixed on the horizontal plane in the point of the tube axis. Then solid angle $\omega$ of the translucent part of the diffuser can be calculated as:

$$
\omega=4 \pi \sin ^{2}\left(\frac{\alpha}{2}\right) \quad[\mathrm{sr}]
$$

where $\alpha=\arctan \left(\frac{r}{h}\right) \quad$ [deg]

$r$ is the radius of the translucent part of the diffuser [m]

$h$ - distance between diffuser and sensor on the horizontal plane [m].

The total light transmission efficiency of the guide system depends on the light transmittance of its components. The above mentioned experiments were focus on the study of the daylight transmission throughout the complete system. In this case the measured data of illuminance at the level of the system input and at the level of the system output can be used for calculation of the light transmission efficiency $\eta$ of the sample. Then, value of the incoming luminous flux $\Phi_{e}$. i.e. flux entering the guidance system is:

$$
\Phi_{e}=E_{e} \times S \quad[\operatorname{lm}]
$$

where

$\underline{E_{e}}$ is measured horizontal illuminance at the level of the CMP [lx].

The luminous flux $\Phi_{i}$ leaving the guide output at the level of the diffuser can be calculated applying equations (5) and (6). Then value of the luminous intensity $I$ is:

$$
I=\frac{E_{i}}{h^{2}} \quad \text { [cd] }
$$

where

$E_{i}$ is measured illuminance on the reference plane in the box [lx]

and the luminous flux $\Phi_{i}$ will be: 


$$
\Phi_{i}=I \times \omega \quad[\operatorname{lm}]
$$

Finally, the light transmission s efficiency $\eta$ of the complete guidance system is determined as a ratio of the output to input luminous flux:

$$
\eta=100 \frac{\Phi_{i}}{\Phi_{e}}
$$

\section{Daylight Luminous Efficiency on the Investigated Light Guide System with the CMP}

The light transmission efficiency as example was expressed for selected fragments of the daily illuminance courses. Data were recorded on $25^{\text {th }}$ May, 2015 at the level of the CMP, Figure 6, and simultaneously on the reference plane in the box, Figure 7. Using radius of the tube $r=0.26 \mathrm{~m}$, distance between diffuser and reference plane $h=0.75 \mathrm{~m}$ and formulae (1) - (7) the l light transmission efficiencies $\eta$ of the guidance system consisting of the CMP head, $0.6 \mathrm{~m}$ length hollow tube and translucent diffuser were calculated. Results with average values of $\eta$ are summarised in Table 2. Results indicate that light transmission efficiency of the guidance system with the CMP head is very sensitive for angle of incident rays. It was found value $\eta=2.49 \%$.

If incident direct solar beams are not concentrated in the focus of the parabolic mirror, it can occur situation that high quantity of incident luminous flux can be reflected back to the space as it is shown in the Figure 1. The input of the hollow tube is in the axis shaded by the small metallic concave element which results into reduction of the system efficiency under sunless skies when only diffuse light is transmitted.

Light guidance systems equipped with the CMP head require tracing facilities to direct parabolic mirror towards the sun position and then as much as direct sunlight can be collected.

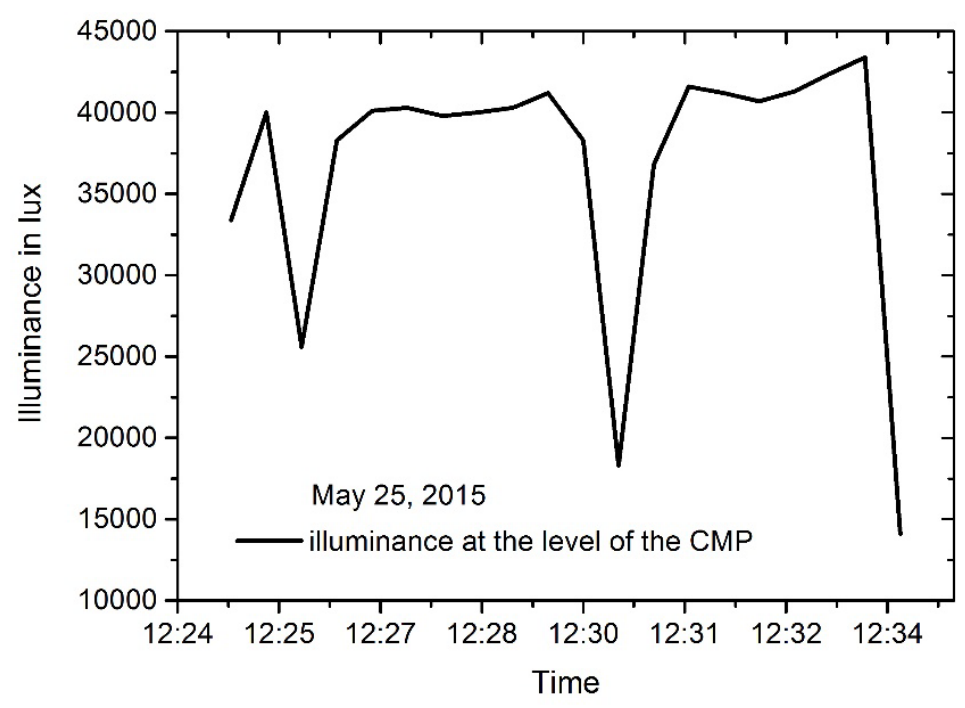

Figure. 6 The daylight illuminance at the level of the CMP, May 25, 2015 


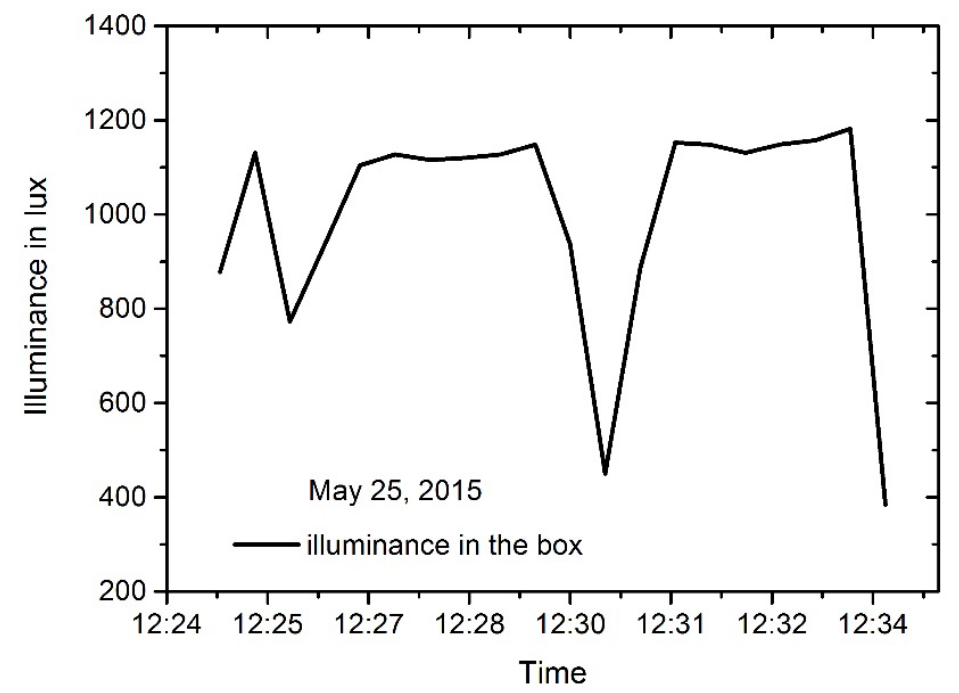

Figure. 7 The daylight illuminance in the box, May 25, 2015

Table. 2 Light transmission efficiency of the guidance system with the CMP, May 24, 2015

\begin{tabular}{|c|c|c|c|c|c|}
\hline \multirow{3}{*}{ Time } & \multirow{2}{*}{\multicolumn{2}{|c|}{ Horizontal illuminance }} & \multicolumn{2}{|c|}{ Luminous flux } & \multirow{3}{*}{$\begin{array}{c}\begin{array}{c}\text { Luminous } \\
\text { efficiency }\end{array} \\
\eta \\
\end{array}$} \\
\hline & & & \multirow{2}{*}{$\begin{array}{c}\text { In the output } \\
\Phi_{i}\end{array}$} & \multirow{2}{*}{$\begin{array}{c}\text { In the input } \\
\Phi_{e} \\
\end{array}$} & \\
\hline & $E_{i}$ & $E_{e}$ & & & \\
\hline hour & {$[\mathrm{lx}]$} & {$[\mathrm{lx}]$} & {$[\operatorname{lm}]$} & {$[\mathrm{lm}]$} & {$[\%]$} \\
\hline $12: 25: 00$ & 878 & 33400 & 171.18 & 7093.21 & 2.413 \\
\hline $12: 25: 30$ & 1131 & 40000 & 220.51 & 8494.87 & 2.596 \\
\hline $12: 26: 00$ & 773 & 25600 & 150.71 & 5436.71 & 2.772 \\
\hline 12:26:30 & 937 & 38300 & 182.68 & 8133.83 & 2.246 \\
\hline $12: 27: 00$ & 1104 & 40100 & 215.24 & 8516.10 & 2.527 \\
\hline $12: 27: 30$ & 1127 & 40300 & 219.73 & 8558.58 & 2.567 \\
\hline 12:28:00 & 1116 & 39800 & 217.58 & 8452.39 & 2.574 \\
\hline $12: 28: 30$ & 1120 & 40000 & 218.36 & 8494.87 & 2.571 \\
\hline $12: 29: 00$ & 1127 & 40300 & 219.73 & 8558.58 & 2.567 \\
\hline $12: 29: 30$ & 1148 & 41200 & 223.82 & 8749.71 & 2.558 \\
\hline 12:30:00 & 937 & 38300 & 182.68 & 8133.83 & 2.246 \\
\hline $12: 30: 30$ & 450 & 18300 & 87.73 & 3886.40 & 2.257 \\
\hline 12:31:00 & 886 & 36800 & 172.74 & 7815.28 & 2.210 \\
\hline $12: 31: 30$ & 1152 & 41600 & 224.60 & 8834.66 & 2.542 \\
\hline $12: 32: 00$ & 1148 & 41200 & 223.82 & 8749.71 & 2.558 \\
\hline $12: 32: 30$ & 1131 & 40700 & 220.51 & 8643.53 & 2.551 \\
\hline 12:33:00 & 1149 & 41300 & 224.01 & 8770.95 & 2.554 \\
\hline 12:33:30 & 1157 & 42400 & 225.57 & 9004.56 & 2.505 \\
\hline 12:34:00 & 1182 & 43400 & 230.45 & 9216.93 & 2.500 \\
\hline \multirow[t]{2}{*}{$12: 34: 30$} & 384 & 14100 & 74.87 & 2994.44 & 2.500 \\
\hline & & & & Average & 2.491 \\
\hline
\end{tabular}

\section{Conclusion}

The light transmission efficiency of the sample of the hollow light guide prototype with the CMP head was experimentally investigated in the laboratory conditions. This system was installed in the stable position with 
orientation of the parabolic mirror towards zenith. Two sources of daylight occur in the nature: sun producing direct sun beams with very high intensity and sky producing diffuse light of the lower intensity.

The idea of the CMP head construction is to focus direct beams by parabolic mirror and redirect them towards the highly reflective tube of the light guide. Experiments prove that if parabolic mirror is not tracking to the sun position only a limited part of sun rays affecting the light guide head is redirected into the tube. When the CMP head is illuminated by the diffuse light the light transmission efficiency can be expected in the level of $2.5 \%$. The value of the light transmission efficiency was determined from recorded illuminance data.

The light transmission efficiency is very low which indicates the fact that without the tracking system the advantage of this light guiding system is not used properly in the conditions of temperate climatic region. The passive installation of the light guide prototype in the static position could be applied only in regions with hot climate and with high solar altitudes. The light transmission efficiency of the system is increased significantly in the case of the tracking of the parabolic mirror to the intensive solar radiation position. An automatic operation of the tracking is recommended for the practical installations in buildings.

\section{Acknowledgements}

This paper has been worked out under the project No. LO1408 "AdMaS UP - Advanced Materials, Structures and Technologies", supported by Ministry of Education, Youth and Sports under the "National Sustainability Programme I" of Czech Republic, project APVV-0118-12 and VEGA 2/0042/17.

\section{References}

[1] CIE 173 (2006) Tubular daylight guidance systems. Technical Report. Vienna: CIE Central Bureau.

[2] Carter, J. (2004) Developments in tubular daylight guidance systems. Building Research Information, 32(3),220-234.

[3] Edmonds, I.R., Moore, G.I., Smith G.B., Swift, P.D. (1995) Daylighting enhancement with light pipes coupled to laser-cut light-deflecting panels. Lighting Research Technology, 27, 27-35.

[4] Aizenberg, J.B. (2013) Hollow light guides: 50 Years of research, development, manufacture and application - a retrospective and looking to the future. Light and Engineering, 21(4), 21-25.

[5] Edmonds, I. (2010) Transmission of mirror light pipes with triangular, rectangular, rhombic and hexagonal cross section. Solar Energy, 84(6), 928-938.

[6] García-Fernández, B., Vázquez-Molini, D., Fernández-Balbuena, A.A. (2011) Lighting quality for aluminum and prismatic light guides. Proc. SPIE 8170, Illumination Optics II, 81700T, 2011.

[7] Jong-Woei Whang, A., Lin, C.M., Yeh, S.C. (2013) Investigation of prismatic daylight collectors with different apexes. Transactions of the ASME, Journal of Solar Energy Engineering, 135(1), art. no. 11015.

[8] Phillips, D. (2004) Daylighting: Natural Light in Architecture. Oxford: Architectural Press, Elsevier.

[9] Carter, D.J. (2002) The measured and predicted performance of passive solar light pipe systems. Lighting Research Technology, 33(1), 39-52.

[109] Oakley, G., Riffat, S.B., Shao, L. (2000) Daylight performance of light pipes. Solar Energy, 69(2), 89-98.

[11] Shao, L., Riffat, S.B., Hicks, W., Yohannes, I. (1997) A study of performance of light pipes under cloudy and sunny conditions in the UK. Right Light, 1(4), 155-159.

[12] Shao, L., Elmualin, A.A., Yohannes, I. (1998) Mirror light pipes daylighting performance in real buildings. Lighting Research Technology, 30(3), 37-40.

[13] Marwaee, A., Carter, D.J. (2006) A field study of tubular daylight guidance installations. Lighting Research Technology, 38(3), 241-58.

[14] Chella, F., Zazzini, P., Carta, G. (2006) Compared numerical and reduced scale experimental analysis on light pipes performances. In. $5^{\text {th }}$ International Conference on Sustainable Energy Technologies SET 2006. Vicenza, 263-268.

[15] Kocifaj, M., Darula, S., Kittler, R. (2008) Holigilm: hollow light guide interior illumination method - an analytic calculation approach for cylindrical light-tubes. Solar Energy, 82(3), 247-59.

[16] Su, Y., Khan, N., Riffat, S.B., Oakley, G. (2012) Comparative monitoring and data regression of various sized commercial light pipes. Energy and Buildings, 50, 308-314.

[17] Darula, S., Kittler, R. (2009) Measurements of optical properties of hollow light guide components using sky simulations. In: Proc. of Int. Conf. Light - Svetlo 2009, Bratislava: SSTS, 74-77.

[18] Light pipe, www.lightway. Cz, (18/12/2017). 